\title{
Constant round distributed domination on graph classes with bounded expansion
}

\author{
Simeon Kublenz ${ }^{1}$, Sebastian Siebertz ${ }^{1[0000-0002-6347-1198]}$, and \\ Alexandre Vigny ${ }^{1[0000-0002-4298-8876]}$ \\ University of Bremen, Bremen, Germany \\ $\{k u b l e n z$, siebertz, vigny\}@uni-bremen. de
}

\begin{abstract}
We show that the dominating set problem admits a constant factor approximation in a constant number of rounds in the LOCAL model of distributed computing on graph classes with bounded expansion. This generalizes a result of Czygrinow et al. for graphs with excluded topological minors.
\end{abstract}

We correct in error in Lemma 1 in the conference version of this paper.

Keywords: Dominating set $\cdot$ LOCAL algorithm - Bounded expansion graph classes.

\section{Introduction}

A dominating set in an undirected and simple graph $G$ is a set $D \subseteq V(G)$ such that every vertex $v \in V(G)$ either belongs to $D$ or has a neighbor in $D$. The Minimum Dominating SeT problem takes as input a graph $G$ and the objective is to find a minimum size dominating set of $G$. The decision problem whether a graph admits a dominating set of size $k$ is NP-hard [23] and this even holds in very restricted settings, e.g. on planar graphs of maximum degree 3 [17].

Consequently, attention shifted from computing exact solutions to approximating near optimal dominating sets. The simple greedy algorithm computes an $\ln n$ approximation (where $n$ is number of vertices of the input graph) of a minimum dominating set $[21,29]$, and for general graphs this algorithm is near optimal - it is NP-hard to approximate minimum dominating sets within factor $(1-\epsilon) \ln n$ for every $\epsilon>0$ [10].

Therefore, researchers tried to identify restricted graph classes where better (sequential) approximations are possible. The problem admits a PTAS on classes with subexponential expansion [19]. Here, expansion refers to the edge density of bounded depth minors, which we will define in detail below. Important examples of classes with subexponential expansion include the class of planar graphs and more generally classes that exclude some fixed graph as a minor. The dominating set problem admits a constant factor approximation on classes of bounded degeneracy (equivalently, of bounded arboricity) [5,28] and an $\mathcal{O}(\ln \gamma)$ approximation (where $\gamma$ denotes the size of a minimum dominating set) on classes of bounded VC-dimension $[7,14]$. In fact, the greedy algorithm can be modified to 
yield a constant factor approximation on graphs with bounded degeneracy [22] and an $\mathcal{O}(\ln \gamma)$ approximation on biclique-free graphs (graphs that exclude some fixed complete bipartite graph $K_{t, t}$ as a subgraph) [33]. However, it is unlikely that polynomial-time constant factor approximations exist even on $K_{3,3}$-free graphs [33]. The general goal in this line of research is to identify the broadest graph classes on which the dominating set problem (or other important problems that are hard on general graphs) can be approximated efficiently with a certain guarantee on the approximation factor. These limits of tractability are often captured by abstract notions, such as expansion, degeneracy or VC-dimension of graph classes.

In this paper we study the distributed time complexity of finding dominating sets in the classic LOCAL model of distributed computing, which can be traced back at least to the seminal work of Gallager, Humblet and Spira [16]. In this model, a distributed system is modeled by an undirected (connected) graph $G$, in which every vertex represents a computational entity of the network and every edge represents a bidirectional communication channel. The vertices are equipped with unique identifiers. In a distributed algorithm, initially, the nodes have no knowledge about the network graph. They must then communicate and coordinate their actions by passing messages to one another in order to achieve a common goal, in our case, to compute a dominating set of the network graph. The LOCAL model focuses on the aspects of communication complexity and therefore the main measure for the efficiency of a distributed algorithm is the number of communication rounds it needs until it returns its answer.

Kuhn et al. [25] proved that in $r$ rounds on an $n$-vertex graphs of maximum degree $\Delta$ one can approximate minimum dominating sets only within a factor $\Omega\left(n^{c / r^{2}} / r\right)$ and $\Omega\left(\Delta^{1 /(r+1)} / r\right)$, respectively, where $c$ is a constant. This implies that, in general, to achieve a constant approximation ratio, we need at least $\Omega(\sqrt{\log n / \log \log n})$ and $\Omega(\log \Delta / \log \log \Delta)$ communication rounds, respectively. Kuhn et al. [25] also presented a $(1+\epsilon) \ln \Delta$-approximation in that runs in $\mathcal{O}(\log (n) / \epsilon)$ rounds for any $\epsilon>0$, Barenboim et al. [6] presented a deterministic $\mathcal{O}\left((\log n)^{k-1}\right)$-time algorithm that provides an $\mathcal{O}\left(n^{1 / k}\right)$-approximation, for any integer parameter $k \geqslant 2$. More recently, the combined works of Rozhon, Ghaffari, Kuhn, and Maus $[18,32]$ provide an algorithm computing a $(1+\epsilon)$-approximation of the dominating set in poly $(\log (n) / \epsilon)$ rounds [32, Corollary 3.11].

For graphs of degeneracy a (equivalent to arboricity up to factor 2), Lenzen and Wattenhofer [28] provided an algorithm that achieves a factor $\mathcal{O}\left(a^{2}\right)$ approximation in randomized time $\mathcal{O}(\log n)$, and a deterministic $\mathcal{O}(a \log \Delta)$ approximation algorithm with $\mathcal{O}(\log \Delta)$ rounds. Graphs of bounded degeneracy include all graphs that exclude a fixed graph as a (topological) minor and in particular, all planar graphs and any class of bounded genus.

Amiri et al. [1] provided a deterministic $\mathcal{O}(\log n)$ time constant factor approximation algorithm on classes of bounded expansion (which extends also to connected dominating sets). Czygrinow et al. [8] showed that for any given $\epsilon>0$, 
$(1+\epsilon)$-approximations of a maximum independent set, a maximum matching, and a minimum dominating set, can be computed in $\mathcal{O}\left(\log ^{*} n\right)$ rounds in planar graphs, which is asymptotically optimal [27].

Lenzen et al. [26] proposed a constant factor approximation on planar graphs that can be computed in a constant number of communication rounds (see also [35] for a finer analysis of the approximation factor). Wawrzyniak [34] showed that message sizes of $\mathcal{O}(\log n)$ suffice to give a constant factor approximation on planar graphs in a constant number of rounds. In terms of lower bounds, Hilke et al. [20] showed that there is no deterministic local algorithm (constant-time distributed graph algorithm) that finds a $(7-\epsilon)$-approximation of a minimum dominating set on planar graphs, for any positive constant $\epsilon$.

The results for planar graphs were gradually extended to classes with bounded genus [2,3], classes with sublogarithmic expansion [4] and eventually by Czygrinow et al. [9] to classes with excluded topological minors. Again, one of the main goals in this line of research is to find the most general graph classes on which the dominating set problem admits a constant factor approximation in a constant number of rounds.

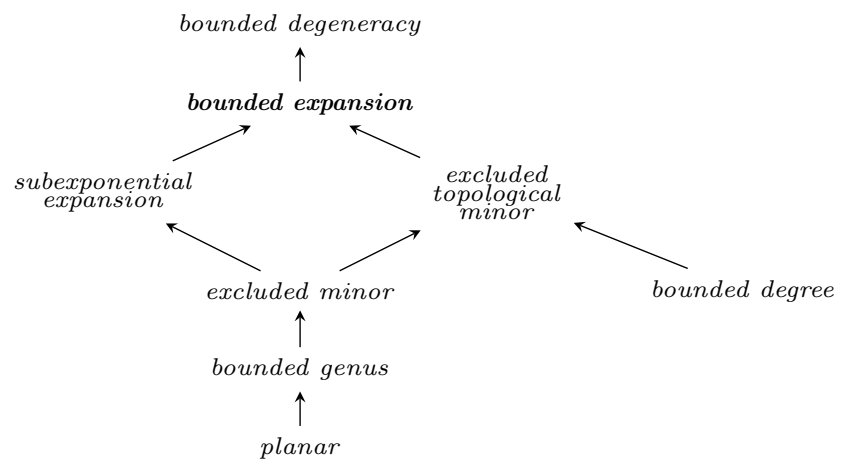

Fig. 1. Inclusion diagram of the mentioned graph classes.

We take a step towards this goal and generalize the result of Czygrinow et al. [9] to classes of bounded expansion. The notion of bounded expansion was introduced by Nešetřil and Ossona de Mendez [30] and offers an abstract definition of uniform sparseness in graphs. It is based on bounding the density of shallow minors. Intuitively, while a minor is obtained by contracting arbitrary connected subgraphs of a graph to new vertices, in an $r$-shallow minor we are only allowed to contract connected subgraphs of radius at most $r$.

A class of graphs has bounded expansion if for every radius $r$ the set of all $r$-shallow minors has edge density bounded by a constant depending only on $r$. We write $\nabla_{r}(G)$ for the maximal edge density of an $r$-shallow minor of a graph $G$. Of course, every class $\mathscr{C}$ that excludes a fixed graph $H$ as a minor has bounded expansion. For such classes there exists an absolute constant $c$ such that for all 
$G \in \mathscr{C}$ and all $r$ we have $\nabla_{r}(G) \leqslant c$. Special cases are the class of planar graphs, every class of graphs that can be drawn with a bounded number of crossings, and every class of graphs that embeds into a fixed surface. Every class of intersection graphs of low density objects in low dimensional Euclidean space has polynomial expansion, that is, the function $\nabla_{r}$ is bounded polynomially in $r$ on $\mathscr{C}$. Also every class $\mathscr{C}$ that excludes a fixed graph $H$ as a topological minor has bounded expansion. Important special cases are classes of bounded degree and classes of graphs that can be drawn with a linear number of crossings Further examples include classes with bounded queue-number, bounded stack-number or bounded non-repetitive chromatic number and the class of Erdös-Rényi random graphs with constant average degree $d / n, G(n, d / n)$, has asymptotically almost surely bounded expansion. See $[19,31]$ for all these examples.

Hence, classes of bounded expansion are much more general than classes excluding a topological minor. On the other hand, maybe not surprisingly, when performing local computations, it is not properties of minors or topological minors, but rather of shallow minors that allow the necessary combinatorial arguments in the algorithms. This observation was already made in the study of the kernelization complexity of dominating set on classes of sparse graphs $[11,12,13,15,24]$. On the other hand, degenerate classes are those classes where only $\nabla_{0}(G)$ is bounded. These classes are hence more general than classes of bounded expansion. See Fig. 1 for an inclusion diagram of the mentioned classes.

The algorithm of Czygrinow et al. [9] is based on an quite complicated iterative process of choosing dominating vertices from so called pseudo-covers. Based on the fact that classes with excluded topological minors in particular exclude some complete bipartite graph $K_{t, t}$ as a subgraph it is proved that this iterative process terminates after at most $t$ rounds and produces a good approximation of a minimum dominating set.

In this paper we make three contributions. First, we simplify the arguments used by Czygrinow et al. and give a much more accessible description of their algorithm. Second, we identify the property that $\nabla_{1}(G)$ is bounded by a constant as the key property that makes the algorithm work. Classes with only this restriction are even more general than bounded expansion classes, hence, we generalize the algorithm to the most general classes on which it (and similar approaches based on covers or pseudo-covers) can work. We demonstrate that the pseudo-covering method cannot be extended e.g. to classes of bounded degeneracy. Finally, Czygrinow et al. explicitly stated that they did not aim to optimize any constants, and as presented, the constants in their construction are enormous. We optimize the bounds that arise in the algorithm in terms of $\nabla_{1}(G)$. Even though the constants are still large, they are by magnitudes smaller than those in the original presentation.

Theorem 1. There exists a LOCAL algorithm that for any given graph $G$ and an upper bound on $\nabla_{1}(G)$ as input computes in a constant number of rounds a dominating set of size $\mathcal{O}\left(\nabla_{1}(G)^{4 t \nabla_{1}(G)+t}\right) \cdot \gamma(G)$, where $t \leqslant 2 \nabla_{1}(G)+1$ is minimum such that $K_{t, t} \nsubseteq G$. 
Before we go into the technical details let us give an overview of the algorithm. The algorithm works in three steps, in each step $(i \in\{1,2,3\})$ computing a small set $D_{i}$ that is added to the dominating set.

1. Compute the set $D_{1}$ of all $v$ such that $N(v)$ cannot be dominated by a small number (the constant $2 \nabla_{1}(G)$ ) of vertices different from $v$. Remove $D_{1}$ from $G$ and mark all its neighbors as dominated. The fact that $\left|D_{1}\right|$ is linearly bounded in $\gamma(G)$ goes back to work of [26] and we prove our bounds in Lemma 1.

2. In parallel for every vertex $v=v_{1}$ we compute all so called domination sequences $v_{1}, \ldots, v_{s}$, defined formally in Definition 3 . This step is based on the construction of pseudo-covers as in the work of Czygrinow et al. [9]. We add all vertices $v_{s}$ to the set $D_{2}$. We prove that this set is small compared to $\gamma(G)$ in Lemma 10. Remove $D_{2}$ from $G$ and mark its neighbors as dominated.

3. All remaining vertices have small degree, as proved in Corollary 2, and hence in a final step we can add all non-dominated vertices to a set $D_{3}$. We finally return the set $D_{1} \cup D_{2} \cup D_{3}$.

The main open question that remains in this line of research is whether we can compute constant factor approximations of minimum dominating sets in a constant number of rounds in classes of bounded degeneracy.

\section{Preliminaries}

In this section we fix our notation and prove some basic lemmas required for the algorithm. We use standard notation from graph theory and refer to the literature for extensive background. For an undirected and simple graph $G$ we denote by $V(G)$ the vertex set and by $E(G)$ the edge set of $G$. We also refer to the literature, for the formal definition of the LOCAL model of distributed computing.

A graph $H$ is a minor of a graph $G$, written $H \preceq G$, if there is a set $\left\{G_{v}: v \in V(H)\right\}$ of pairwise vertex disjoint and connected subgraphs $G_{v} \subseteq G$ such that if $\{u, v\} \in E(H)$, then there is an edge between a vertex of $G_{u}$ and a vertex of $G_{v}$. We call $V\left(G_{v}\right)$ the branch set of $v$ and say that it is contracted to the vertex $v$.

For a non-negative integer $r$, a graph $H$ is an $r$-shallow minor of $G$, written $H \unlhd_{r} G$, if there is a set $\left\{G_{v}: v \in V(H)\right\}$ of pairwise vertex disjoint connected subgraphs $G_{v} \subseteq G$ of radius at most $r$ such that if $\{u, v\} \in E(H)$, then there is an edge between a vertex of $G_{u}$ and a vertex of $G_{v}$. Observe that a 0 -shallow minor of $G$ is just a subgraph of $G$.

We write $\nabla_{r}(G)$ for $\max _{H \leq_{r} G}|E(H)| /|V(H)|$. Observe that $\nabla_{0}(G)$ denotes the maximum average edge density of $G$ and $2 \nabla_{0}(G)$ bounds the degeneracy of $G$, which is defined as $\max _{H \subseteq G} \delta(H)$. Here, $\delta(H)$ denotes the minimum degree of $H$. A class $\mathscr{C}$ of graphs has bounded expansion if there is a function $f: \mathbb{N} \rightarrow \mathbb{N}$ such that $\nabla_{r}(G) \leqslant f(r)$ for all graphs $G \in \mathscr{C}$. This is equivalent to demanding that the degeneracy of each $r$-shallow minor of $G$ is functionally bounded by $r$. 
We write $K_{s, t}$ for the complete bipartite graph with partitions of size $s$ and $t$, respectively. Observe that $K_{t, t}$ has $2 t$ vertices and $t^{2}$ edges, hence, if $\nabla_{0}(G)<t / 2$, then $G$ excludes $K_{t, t}$ as a subgraph.

For a graph $G$ and $v \in V(G)$ we write $N(v)=\{w:\{v, w\} \in E(G)\}$ for the open neighborhood of $v$ and $N[v]=N(v) \cup\{v\}$ for the closed neighborhood of $v$. For a set $A \subseteq V(G)$ let $N[A]=\bigcup_{v \in A} N[v]$. We write $N_{r}[v]$ for the set of vertices at distance at most $r$ from a vertex $v$. A dominating set in a graph $G$ is a set $D \subseteq V(G)$ such that $N[D]=V(G)$. We write $\gamma(G)$ for the size of a minimum dominating set of $G$. For $W \subseteq V(G)$ we say that a set $Z \subseteq V(G)$ dominates or covers or is a cover of $W$ if $W \subseteq N[Z]$. Observe that we do not require $Z \cap W=\varnothing$ as Czygrinow et al. do for covers.

The following lemma is one of the key lemmas used for the algorithm. It goes back to [26].

Lemma 1. Let $G$ be a graph. Then there are less than $\left(\nabla_{1}(G)+2\right) \gamma(G)$ vertices $v$ with the property that $N(v)$ cannot be dominated by at most $2 \nabla_{1}(G)$ vertices different from $v$.

Proof. Let $\gamma=\gamma(G)$ and $\nabla_{1}=\nabla_{1}(G)$ and assume that there are $\left(\nabla_{1}+2\right) \gamma$ such vertices $a_{1}, \ldots, a_{\left(\nabla_{1}+2\right) \gamma}$. We proceed towards a contradiction. Let $\left\{d_{1}, \ldots, d_{\gamma}\right\}$ be a minimum dominating set. At least $\gamma$ of the $a_{i}$ 's are not in this dominating set. We can hence assume w.l.o.g. that $\left\{a_{1}, \ldots, a_{\left(\nabla_{1}+1\right) \gamma}\right\}$ and $\left\{d_{1}, \ldots, d_{\gamma}\right\}$ are two disjoint sets of vertices.

We build a 1-shallow minor $H$ of the graph $G$ with the following $\left(\nabla_{1}+2\right) \gamma$ branch sets. For every $i \leqslant\left(\nabla_{1}+1\right) \gamma$, we have a branch set $A_{i}=\left\{a_{i}\right\}$ and a branch set $D_{i}=N\left[d_{i}\right] \backslash\left(\left\{a_{1}, \ldots, a_{\left(\nabla_{1}+1\right) \gamma}\right\} \cup \bigcup_{j<i} N\left[d_{j}\right] \cup\left\{d_{i+1}, \ldots, d_{\gamma}\right\}\right)$. We call the associated vertices of $H a_{1}^{\prime}, \ldots, a_{\left(\nabla_{1}+1\right) \gamma}^{\prime}, d_{1}^{\prime}, \ldots, d_{\gamma}^{\prime}$.

We now count the edges of $H$. Since $\left\{d_{1}, \ldots, d_{\gamma}\right\}$ is a dominating set of $G$ and by assumption on $N\left(a_{i}\right)$, we have that in $H$, every $a_{i}^{\prime}$ has degree at least $2 \nabla_{1}+1$. The number of edges having both end points among the $a_{i}^{\prime}$ is bounded by $\left(\nabla_{1}+1\right) \gamma \nabla_{0}(G)$, end hence by $\left(\nabla_{1}+1\right) \gamma \nabla_{1}$. We therefore get that $\left|E_{H}\right| \geqslant$ $\left(\nabla_{1}+1\right) \gamma\left(2 \nabla_{1}+1\right)-\left(\nabla_{1}+1\right) \gamma \nabla_{1} \geqslant\left(\nabla_{1}+1\right)^{2} \gamma$. Since $\left|V_{H}\right|=\left(\nabla_{1}+2\right) \gamma$, we have that $\frac{\left|E_{H}\right|}{\left|V_{H}\right|} \geqslant \frac{\left(\nabla_{1}+1\right)^{2} \gamma}{\left(\nabla_{1}+2\right) \gamma} \geqslant \frac{\left(\nabla_{1}+1\right)^{2}}{\left(\nabla_{1}+2\right)}>\nabla_{1}$, a contradiction.

Note that we cannot locally determine the number $\nabla_{1}(G)$. We must hence assume that it is given with the input. Observe that similarly, the algorithm of Czygrinow et al. works with the assumption that the input excludes a complete graph with $t$ vertices as a topological minor. This implies a bound on the edge density of topological minors in $G$, which can be seen as being given with the input.

The algorithm proceeds in three phases. The first phase is based on Lemma 1 as follows. In the LOCAL model we can learn the distance-2 neighborhood $N_{2}[v]$ of every vertex $v$ in 2 rounds, and then locally check whether $N(v)$ can be dominated by at most $2 \nabla_{1}(G)$ vertices. 
We let $D_{1}$ be the set of all vertices that do not have this property. By Lemma 1 we have $\left|D_{1}\right| \leqslant\left(\nabla_{1}(G)+2\right) \gamma(G)$. We remove $D_{1}$ from the graph and mark all its neighbors as dominated in one additional round.

In the following we fix a graph $G$ and we assume that $N(v)$ can be dominating by at most $2 \nabla_{1}(G)$ vertices different from $v$ for all $v \in V(G)$. We write $\nabla_{1}$ for $\nabla_{1}(G)$ and we let $t \leqslant 2 \nabla_{0}(G)+1$ be the smallest positive integer such that $G$ excludes $K_{t, t}$ as a subgraph. Note that this number is not required as part of the input. We let $k:=2 \nabla_{1}$.

Example 1. A planar $n$-vertex graph has at most $3 n-6$ edges. A minor of a planar graph is again planar, hence for planar graphs $G$ we have $\nabla_{r}(G) \leqslant 3$ for all $r \geqslant 0$ and $k=2 \nabla_{1}(G) \leqslant 6$.

We also fix a minimum dominating set $D$ of $G$ of size $\gamma$. The following lemma is proved exactly as Lemma 1.

Lemma 2. There are less than $\left(\nabla_{1}+2\right) \gamma$ vertices $v$ with the property that $N(v)$ cannot be dominated by at most $2 \nabla_{1}$ vertices from $D$ and different from $v$.

Unfortunately, we cannot determine these vertices locally, as it requires knowledge of $D$, however, this structural property is very useful for our further argumentation.

Denote by $\hat{D}$ the set of all vertices $v$ whose neighborhood cannot be dominated by $2 \nabla_{1}$ vertices of $D$ different from $v$. Let $D^{\prime}=D \cup \hat{D}$.

According to Lemma $2, D^{\prime}$ contains at most $\left(\nabla_{1}+2\right) \gamma$ vertices. Let us stress that $D^{\prime}$ will never be computed by our LOCAL algorithm. We only use its existence in the correctness proofs.

We can apply these lemmas to obtain a constant factor approximation for a dominating set only if $\nabla_{1}(G)$ is bounded by a constant. For example in graphs of bounded degeneracy in general the number of vertices that dominate the neighborhood of a vertex can only be bounded by $\gamma(G)$. Hence, the approach based on covers and pseudo-covers that is employed in the following cannot be extended to degenerate graph classes.

Example 2. Let $G(\gamma, m)$ be the graph with vertices $v_{i}$ for $1 \leqslant i \leqslant \gamma, w^{j}$ for $1 \leqslant j \leqslant m$ and $s_{i}^{j}$ for $1 \leqslant i \leqslant \gamma, 1 \leqslant j \leqslant m$ (see Figure 2). We have the edges $\left\{v_{1}, w^{j}\right\}$ for $1 \leqslant j \leqslant m$, hence $v_{1}$ dominates all $w^{j}$. We have the edges $\left\{w^{j}, s_{i}^{j}\right\}$ for all $1 \leqslant i \leqslant \gamma, 1 \leqslant j \leqslant m$, hence, the $s_{i}^{j}$ are neighbors of $w^{j}$. Finally, we have the edges $\left\{v_{i}, s_{i}^{j}\right\}$, that is, $v_{i}$ dominates the $i$ th neighbor of $w^{j}$. Hence, for $m>\gamma, G(\gamma, m)$ has a dominating set of size $\gamma$ and $m$ vertices whose neighborhood can be dominated only by $\gamma(G)$ vertices. Lemma 1 implies that $\gamma<2 \nabla_{1}$, and as we can choose $m$ arbitrary large, we cannot usefully apply Lemma 1. Furthermore, $G(\gamma, m)$ is 2-degenerate, showing that these methods cannot be applied on degenerate graph classes. 


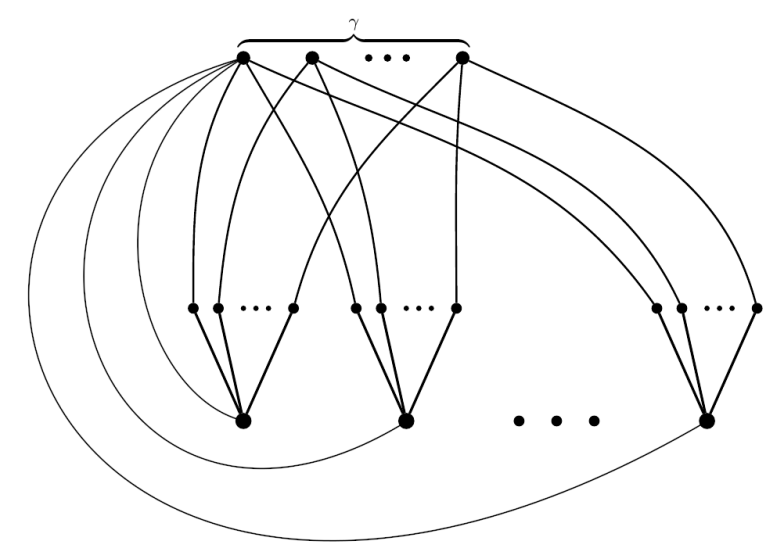

Fig. 2. A 2-degenerate graph where for many $v \in V(G)$ the set $N(v)$ can only be dominated by at least $\gamma$ vertices different from $v$.

\section{Covers and pseudo-covers}

Intuitively, the vertices from a cover of a set $W$ can take different roles. A few vertices of a cover may cover almost the complete set $W$, while a few others are only there to cover what was left over. The key observation of Czygrinow et al. is that in classes that exclude some $K_{t, t}$ as a subgraph, there can only be few of such high degree covering vertices, while there can be arbitrarily many vertices that cover at most $t-1$ vertices of $W$ (the same vertices can be covered over and over again). This observation can be applied recursively and is distilled into the following two definitions. Recall that by the processing carried out in the first phase of the algorithm we know that every neighborhood $N(v)$ can be covered by $k=2 \nabla_{1}$ vertices different from $v$. We recall all fixed parameters for easy to find reference.

- $G$ : fixed graph.

$-\gamma: \gamma(G)$.

$-\nabla_{1}: \nabla_{1}(G)$.

- $t \quad$ : smallest integer such that $G$ excludes $K_{t, t}$ as a subgraph

- $D_{1}$ : defined and computed in Lemma 1.

- $D$ : fixed dominating set of $G$ of size $\gamma$ (not computed).

- $\hat{D}$ : defined in Lemma 2 (not computed).

- $D^{\prime}: D \cup \hat{D}$ (not computed). 
Following the presentation of [9], we name and fix these constants for the rest of this article.

$-k:=2 \nabla_{1}$.

- $\alpha:=1 / k$.

$-\ell:=8 \nabla_{1} / \alpha^{2}+1=4 k^{3}+1$.

- $q:=4 k^{4}$.

Definition 1. A vertex $z \in V(G)$ is $\alpha$-strong for a vertex set $W \subseteq V(G)$ if $|N[z] \cap W| \geqslant \alpha|W|$.

The following is the key definition by Czygrinow et al. [9].

Definition 2. A pseudo-cover (with parameters $\alpha, \ell, q, k$ ) of a set $W \subseteq V(G)$ is a sequence $\left(v_{1}, \ldots, v_{m}\right)$ of vertices such that for every $i$ we have

- $\left|W \backslash \bigcup_{j \leqslant m} N\left[v_{j}\right]\right| \leqslant q$,

- $v_{i}$ is $\alpha$-strong for $W \backslash \bigcup_{j<i} N\left[v_{j}\right]$,

$-\left|N\left[v_{i}\right] \cap\left(W \backslash \bigcup_{j<i} N\left[v_{j}\right]\right)\right| \geqslant \ell$,

$-m \leqslant k$.

Intuitively, all but at most $q$ elements of the set $W$ are covered by the $\left(v_{i}\right)_{i \leqslant m}$. Additionally, each element of the pseudo-cover dominates both an $\alpha$-fraction of what remains to be dominated, and at least $\ell$ elements. Note that with our choice of constants, if there are more than $q$ vertices not covered yet, any vertex that covers an $\alpha$-fraction of what remains also covers at least $\ell$ elements.

The next lemma shows how to derive the existence of pseudo-covers from the existence of covers.

Lemma 3. Let $W \subseteq V(G)$ be of size at least $q$ and let $Z$ be a cover of $W$ with $k$ elements. There exists an ordering of the vertices of $Z$ as $z_{1}, \ldots, z_{k}$ and $m \leqslant k$ such that $\left(z_{1}, \ldots, z_{m}\right)$ is a pseudo-cover of $W$.

Proof. We build the order greedily by induction. We order the elements by neighborhood size, while removing the neighborhoods of the previously ordered vertices. More precisely, assume that $\left(z_{1}, \ldots, z_{i}\right)$ have been defined for some $i \geqslant 0$. We then define $z_{i+1}$ as the element that maximizes $\left|N[z] \cap\left(W \backslash \bigcup_{j \leqslant i} N\left[z_{j}\right]\right)\right|$.

Once we have ordered all vertices of $Z$, we define $m$ as the maximal integer not larger than $k$ such that for every $i \leqslant m$ we have:

- $z_{i}$ is $\alpha$-strong for $W \backslash \bigcup_{j<i} N\left[z_{j}\right]$, and

- $\left|N\left[z_{i}\right] \cap\left(W \backslash \bigcup_{j \leqslant i} N\left[z_{j}\right]\right)\right| \geqslant \ell$.

This made sure that $\left(z_{1}, \ldots, z_{m}\right)$ satisfies the last 3 properties of a pseudocover of $W$. It only remains to check the first one. To do so, we define $W^{\prime}:=$ $W \backslash \bigcup_{i \leqslant m} N\left[z_{i}\right]$. We want to prove that $\left|W^{\prime}\right| \leqslant q$. Note that because $Z$ covers $W$, if $m=k$ we have $W^{\prime}=\varnothing$ and we are done. We can therefore assume that $m<k$ 
and $W^{\prime} \neq \varnothing$. Since $Z$ is a cover of $W$, we also know that $\left(z_{m+1}, \ldots z_{k}\right)$ is a cover of $W^{\prime}$, therefore there is an element in $\left(z_{m+1}, \ldots z_{k}\right)$ that dominates at least a $1 / k$ fraction of $W^{\prime}$. Thanks to the previously defined order, we know that $z_{m+1}$ is such element. Since $\alpha=1 / k$, it follows that $z_{m+1}$ is $\alpha$-strong for $W^{\prime}$. This, together with the definition of $m$, we have that $\left|N\left[z_{i}\right] \cap\left(W \backslash \bigcup_{j \leqslant i} N\left[z_{j}\right]\right)\right|<\ell$ meaning that $\left|N\left[z_{m+1}\right] \cap W^{\prime}\right|<\ell$. This implies that $\left|W^{\prime}\right| / k<\ell$. And since $\ell=q / k$, we have $\left|W^{\prime}\right|<q$. Hence, $\left(z_{1}, \ldots, z_{m}\right)$ is a pseudo-cover of $W$.

While there can exist unboundedly many covers for a set $W \subseteq V(G)$, the nice observation of Czygrinow et al. was that the number of pseudo-covers is bounded whenever the input graph excludes some biclique $K_{s, t}$ as a subgraph. We do not state the result in this generality, as it leads to enormous constants. Instead, we focus on the case where small covers exist, that is, on the case where $\nabla_{1}(G)$ is bounded and optimize the constants for this case.

Lemma 4. Let $W \subseteq V(G)$ of size at least $8 \nabla_{1} / \alpha^{2}$. Then there are at most $4 \nabla_{1} / \alpha$ vertices that are $\alpha$-strong for $W$.

Proof. Assume that there is such a set $W$ with at least $c:=4 \nabla_{1} / \alpha$ vertices that are $\alpha$-strong for $W$. We build a 1-shallow minor $H$ of the graph $G$ with $|W|$ branch sets. Each branch set is either a single element of $W$, or a pair $\{w, a\}$, where $w$ is in $W$ and $a$ is an $\alpha$-strong vertex for $W$, connected to $w$, and that is not in $W$. This is obtained by iteratively contracting one edge of an $\alpha$-strong vertex with a vertex of $W$. This is possible because $\alpha|W|>c$, so during the process and for any $\alpha$-strong vertex we can find a connected vertex in $W$ that is not part of any contraction.

Once this is done, we have that $\left|V_{H}\right|=|W|$. For the edges, each of the $\alpha$-strong vertices can account for $\alpha|W|$ many edges. We need to subtract $c^{2}$ from the total as we do not count twice an edge between two strong vertices. Therefore $\left|E_{H}\right| \geqslant c \alpha|W|-c^{2}$. Note also that because $|W| \geqslant 8 \nabla_{1} / \alpha^{2}$, we have that $2 \nabla_{1} \geqslant\left(4 \nabla_{1}\right)^{2} /\left(\alpha^{2}|W|\right)$. All of this together leads to:

$$
\frac{\left|E_{H}\right|}{\left|V_{H}\right|} \geqslant \frac{c \alpha|W|-c^{2}}{|W|} \geqslant 4 \nabla_{1}-\frac{\left(4 \nabla_{1}\right)^{2}}{\alpha^{2}|W|} \geqslant 4 \nabla_{1}-2 \nabla_{1}>\nabla_{1}
$$

This contradicts the definition of $\nabla_{1}$.

This leads quickly to a bound on the number of pseudo-covers.

Lemma 5. For every $W \subseteq V(G)$ of size at least $\ell$, the number of pseudo-covers is bounded by $2\left(4 \nabla_{1}(G) / \alpha\right)^{k}$.

The proof of the lemma is exactly as the proof of Lemma 7 in the presentation of Czygrinow et al. [9], we therefore refrain from repeating it here.

We write $\mathcal{T}(v)$ for the set of all pseudo-covers of $N(v)$ and $\mathcal{P}(v)$ for the set of all vertices that appear in a pseudo-cover of $N(v)$.

Corollary 1. For every $v \in V(G)$ with $|N(v)|>\ell$, we have $|\mathcal{T}(v)| \leqslant 2\left(2 k^{2}\right)^{k}$ and $|\mathcal{P}(v)| \leqslant 2 k\left(2 k^{2}\right)^{k} \leqslant(2 k)^{2 k+1}$. 


\section{Finding dominators}

Recall that by Lemma 2 for every $v \in V(G)$ we can cover $N(v)$ with at most $k$ vertices from $D^{\prime}$. To first gain an intuitive understanding of the second phase of the algorithm, where we construct a set $D_{2} \subseteq V(G)$, let us consider the following iterative procedure.

Fix some $v \in V(G)$. Let $v_{1}=v$ and $B_{1}:=N(v)$ and assume $\left|B_{1}\right| \geqslant k^{t-1}(2 t-1)$. We consider $s$ vertices $v_{1}, v_{2} \ldots, v_{s}$ as follows. Choose as $v_{2}$ an arbitrary vertex different from $v_{1}$ that dominates at least $k^{t-2}(2 t-1)$ vertices of $B_{1}$, that is, a vertex that satisfies $\left|N\left[v_{2}\right] \cap B_{1}\right| \geqslant k^{t-2}(2 t-1)$. Note that any vertex $v_{2}$ that dominate a $1 / k$-fraction of $B_{1}$ can be such vertex, i.e. it is enough for $v_{2}$ to be $\alpha$-strong for $B_{1}$.

Let $B_{2}:=N\left(v_{2}\right) \cap B_{1}$. Observe that we consider the open neighborhood of $v_{2}$ here, hence $B_{2}$ does not contain $v_{2}$. Hence, $\left|B_{2}\right| \geqslant k^{t-2}(2 t-1)-1 \geqslant k^{t-2}(2 t-2)$. We continue to choose vertices $v_{3}, \ldots$ inductively just as above. That is, if the vertices $v_{1}, \ldots, v_{i}$ and sets $B_{1}, \ldots, B_{i} \subseteq V(G)$ have been defined, we choose the next vertex $v_{i+1}$ as an arbitrary vertex not in $\left\{v_{1}, \ldots, v_{i}\right\}$ that dominates at least $k^{t-i-1}(2 t-i)$ vertices of $B_{i}$, that is, a vertex with $\left|N\left[v_{i+1}\right] \cap B_{i}\right| \geqslant k^{t-i-1}(2 t-i)$ and let $B_{i+1}:=N\left(v_{i+1}\right) \cap B_{i}$, of size at least $k^{t-i-1}(2 t-i-1)$.

Lemma 6. Assume $|N(v)| \geqslant k^{t-1} \cdot(2 t-1)$. Let $v_{1}, \ldots, v_{s}$ be a maximal sequence obtained as above. Then $s<t$ and $D^{\prime} \cap\left\{v_{1}, \ldots, v_{s}\right\} \neq \varnothing$.

Proof. Assume that we can compute a sequence $v_{1}, v_{2}, \ldots, v_{t}$. By definition, every $v_{i}$ is connected to every vertices of $B_{t}$. For every $1 \leqslant i \leqslant t$ we have $\left|B_{i}\right| \geqslant k^{t-i}(2 t-i)$ and therefore $\left|B_{t}\right| \geqslant t$. This shows that the two sets $\left\{v_{1}, \ldots, v_{t}\right\}$ and $B_{t}$ form a $K_{t, t}$ as a subgraph of $G$. Since this is not possible, the process must stop having performed at most $t-1$ rounds.

We now turn to the second claim of the lemma. Assume that $v_{1}, v_{2}, \ldots, v_{s}$ is a maximal sequence for some $s<t$. We assume $v_{1} \notin \hat{D}$, otherwise, we are done, as $\hat{D} \subseteq D^{\prime}$. Because $s<t$, we have that $B_{s}$ is not empty. Because $B_{s} \subseteq B_{1}=N\left(v_{1}\right)$, we have that $B_{s}$ can be dominated with at most $k$ elements of $D$ (by definition of $\hat{D}$ ), and in particular by at most $k$ elements of $D^{\prime}$. Therefore, there must be an element $v$ of $D^{\prime}$ that dominates a $1 / k$ fraction of $B_{s}$. If $v$ was not one of the $v_{1}, \ldots, v_{s}$, we could have continued the sequence by defining $v_{s+1}:=v$. Since the sequence is maximal, $v$ must be one of the $v_{1}, \ldots, v_{s}$, which leads to $D^{\prime} \cap\left\{v_{1}, \ldots, v_{s}\right\} \neq \varnothing$.

We aim to carry out this iterative process in parallel for all vertices $v \in V(G)$ with a sufficiently large neighborhood. Of course, in the process we cannot tell when we have encountered the element of $D^{\prime}$. Hence, from the constructed vertices $v_{1}, \ldots, v_{s}$ we will simply choose the element $v_{s}$ into the dominating set. Unfortunately, this approach alone can give us arbitrarily large dominating sets, as we can have many choices for the vertices $v_{i}$, while already $v_{1}$ was possibly optimal. We address this issue by restricting the possible choices for the vertices $v_{i}$. 
Definition 3. For any vertex $v \in V(G)$, a $k$-dominating-sequence of $v$ is a sequence $\left(v_{1}, \ldots, v_{s}\right)$ for which we can define sets $B_{1}, \ldots, B_{s}$ such that:

$-v_{1}=v, B_{1} \subseteq N\left(v_{1}\right)$,

- for every $i \leqslant s$ we have $B_{i} \subseteq N\left(v_{i}\right) \cap B_{i-1}$,

$-\left|B_{i}\right| \geqslant k^{t-i}(2 t-i+(t-i) q)$

- and for every $i \leqslant s$ we have $v_{i} \in \mathcal{P}\left(v_{i-1}\right)$.

A $k$-dominating-sequence $\left(v_{1}, \ldots, v_{s}\right)$ is maximal if there is no vertex $u$ such that $\left(v_{1}, \ldots, v_{s}, u\right)$ is a $k$-dominating-sequence.

Note that this definition requires $|N(v)| \geqslant k^{t-1}(2 t-1+(t-1) q)$. For a vertex $v$ with a not sufficiently large neighborhood, there are no $k$-dominatingsequences of $v$. We show two main properties of these dominating-sequences. First, Lemma 7 shows that a maximal dominating sequence must encounter $D^{\prime}$ at some point. Second, with Lemmas 8 to 10, we show that collecting all "end points" of all maximal dominating sequences results in a set $D_{2}$ of size linear in the size of $D^{\prime}$. While $D^{\prime}$ cannot be computed, we can compute $D_{2}$.

Lemma 7. Let $v$ be a vertex and let $\left(v_{1}, \ldots, v_{s}\right)$ be a maximal $k$-dominatingsequence of $v$. Then $s<t$ and $D^{\prime} \cap\left\{v_{1}, \ldots, v_{s}\right\} \neq \varnothing$.

Proof. The statement $s<t$ is proved exactly as for Lemma 6 .

To prove the second statement we assume, in order to reach a contradiction, that $D^{\prime} \cap\left\{v_{1}, \ldots, v_{s}\right\}=\varnothing$. We have that $B_{s} \subseteq N\left(v_{s}\right)$, and remember that $N\left(v_{s}\right)$ can be dominated by at most $k$ elements of $D^{\prime}$. By Lemma 3, we can derive a pseudo-cover $S=\left(u_{1}, \ldots, u_{m}\right)$ of $N\left(v_{s}\right)$, where $m \leqslant k$ and every $u_{i}$ is an element of $D^{\prime}$. Let $X$ denote the set (of size at most $q$ ) of vertices not covered by $S$. As $S$ contains at most $k$ vertices there must exist a vertex $u$ in $S$ that covers at least a $1 / k$ fraction of $B_{s} \backslash X$. By construction, we have that $\left|B_{s}\right| \geqslant k^{t-s} \cdot(2 t-s+(t-s) q) \geqslant k(t+q)$ because $s<t$. Therefore $\left|B_{s} \backslash X\right| \geqslant k$ and we have

$$
\left|N[u] \cap B_{s}\right| \geqslant \frac{\left|B_{s}\right|-q}{k} \geqslant \frac{k^{t-s}(2 t-s+(t-s) q)-q}{k},
$$

hence

$$
\left|N[u] \cap B_{s}\right| \geqslant \frac{k^{t-s}(2 t-s+(t-s-1) q)}{k} \geqslant k^{t-s-1}(2 t-s+(t-s-1) q),
$$

and therefore

$$
\left|N(u) \cap B_{s}\right| \geqslant\left|N[u] \cap B_{s}\right|-1 \geqslant k^{t-s-1}(2 t-s-1+(t-s-1) q) .
$$

So we can continue the sequence $\left(v_{1}, \ldots, v_{s}\right)$ by defining $v_{s+1}:=u$. In conclusion if $\left(v_{1}, \ldots, v_{s}\right)$ is a maximal sequence, it contains an element of $D^{\prime}$.

The goal of this modified procedure is first to ensure that every maximal sequence contains an element of $D^{\prime}$ and second, to make sure that there are not to many possible $v_{s}$ (which are the elements that we pick for the dominating set). This is illustrated in the following example and formalized right after that. 
Example 3. Consider the case of planar graphs. Since these graph exclude $K_{3,3}$, i.e. $t=3$, we have that every maximal sequence is of length 1 or 2 . For every $v$ of sufficiently large neighborhood we consider every maximal $k$-dominating-sequence $\left(v_{1}, v_{s}\right)$ of $v$. We then add $v_{s}$ to the set $D_{2}$. We want to show that $\left|D_{2}\right|$ is linearly bounded by $\left|D^{\prime}\right|$ and hence by $\gamma(G)$.

If $s=1$, then we have $v_{s} \in D^{\prime}$ and we are good.

If $s=2$, we have two possibilities. If $v_{2}$ is in $D^{\prime}$, we are good. If however, $v_{2}$ is not in $D^{\prime}$, then $v_{1}$ is in $D^{\prime}$. Additionally, $v_{2}$ is in some pseudo-cover $S$ of $v_{1}$, i.e. $v_{2} \in \mathcal{P}\left(v_{1}\right)$.

By Corollary 1 , we have $\left|\mathcal{P}\left(v_{1}\right)\right| \leqslant(2 k)^{2 k+1}$ (and in fact this number is much smaller in the case of planar graphs). Therefore we have $\left|D_{2}\right| \leqslant\left((2 k)^{2 k+1}+1\right)\left|D^{\prime}\right|$.

We generalize the ideas of Example 3, by explaining what a "few possible choices" in the discussion before Definition 3 means.

Lemma 8. For any maximal $k$-dominating-sequence $\left(v_{1}, \ldots, v_{s}\right)$, and for any $i \leqslant s-1$, we have that

$$
\begin{aligned}
& -v_{i+1} \in \mathcal{P}\left(v_{i}\right), \\
& -\left|N\left(v_{i}\right)\right| \geqslant \ell, \text { and } \\
& -\left|\mathcal{P}\left(v_{i}\right)\right| \leqslant(2 k)^{2 k+1} .
\end{aligned}
$$

Proof. By construction $v_{i+1} \in \mathcal{P}\left(v_{i}\right)$, furthermore, $v_{i}$ dominates at least $B_{i}$, and $\left|B_{i}\right| \geqslant k^{t-i}(2 t-i+(t-i) q) \geqslant q>\ell$. We conclude with Corollary 1.

We now for every $v \in V(G)$ compute all maximal $k$-dominating-sequences starting with $v$. Obviously, as every $v_{i}$ in any $k$-dominating-sequences of $v$ dominates some neighbors of $G$, we can locally compute these steps after having learned the 2-neighborhood $N_{2}[v]$ of every vertex in two rounds in the LOCAL model of computation.

For a set $W \subseteq V(G)$ we write $\mathcal{P}(W)=\bigcup_{v \in W} \mathcal{P}(v)$. Remember that the definition of $\mathcal{P}(v)$ requires that $|N(v)|>\ell$. We simply extend the notation with $\mathcal{P}(v)=\varnothing$ if $|N(v)| \leqslant \ell$. We now define

$$
\mathcal{P}^{(1)}(W):=\mathcal{P}(W)
$$

additionally, for $1<i<t$

$$
\mathcal{P}^{(i)}(W):=\mathcal{P}\left(\mathcal{P}^{(i-1)}(W)\right)
$$

and finally, for every $1 \leqslant i \leqslant t$

$$
\mathcal{P}^{(\leqslant i)}(W):=\bigcup_{1 \leqslant j \leqslant i} \mathcal{P}^{(j)}(W)
$$

Using Lemma 8 , for every $k$-dominating-sequence $\left(v_{1}, \ldots, v_{s}\right)$ we have that $v_{s} \in \mathcal{P}^{(\leqslant k)}\left(v_{1}\right)$. More generally, for every $i \leqslant s$, we have that $v_{s} \in \mathcal{P}^{(\leqslant t)}\left(v_{i}\right)$. 
We define $D_{2}$ as the set of all $u \in V(G)$ such that there is some vertex $v \in V(G)$, and some maximal $k$-dominating-sequence $\left(v_{1}, \ldots, v_{s}\right)$ of $v$ with $u=v_{s}$.

This leads to the following lemma.

Lemma 9. $D_{2} \subseteq \mathcal{P}^{(\leqslant t)}\left(D^{\prime}\right)$.

Proof. This uses the observation made above the statement of this lemma, together with Lemma 7.

Note that while we don't know how to compute $D^{\prime}$, this section explained how to compute $D_{2}$ in 2 rounds with the LOCAL model of computation.

Lemma 10. $\left|D_{2}\right| \leqslant(2 k)^{t(2 k+1)} \cdot\left|D^{\prime}\right|$

Proof. Corollary 1 gives us that $|\mathcal{P}(v)| \leqslant 2 k\left(2 k^{2}\right)^{k}$ for every $v \in V(G)$ with $|N(v)|>\ell$. As $\mathcal{P}(W) \leqslant \sum_{v \in W}|\mathcal{P}(v)|$, we have $P(W) \leqslant|W| \cdot(2 k)^{2 k+1}$. A naive induction yields that for every $i \leqslant t$,

$$
\left|\mathcal{P}^{(\leqslant i)}(W)\right| \leqslant c^{i}|W|,
$$

where $c=(2 k)^{2 k+1}$. Hence with this and Lemma 9 we have

$$
\left|D_{2}\right| \leqslant(2 k)^{t(2 k+1)} \cdot\left|D^{\prime}\right|
$$

\section{$5 \quad$ Cleaning up}

We now show that after defining and computing $D_{2}$ as explained in the previous section, every neighborhood is almost entirely dominated by $D_{2}$. More precisely, for every vertex $v$ of the graph $\left|\left\{v^{\prime} \in N(v): v^{\prime} \notin N\left(D_{2}\right)\right\}\right|<k^{t-1}(2 t-1+(t-1) q)$ holds.

Before explaining why this holds, note that it implies that, in particular, the vertices of $D$ have at most $k^{t-1}(2 t-1+(t-1) q)$ non-dominated neighbors. Since every vertex is either in $D$ or a neighbor of some element in $D$, this implies that in the whole graph there are at most $k^{t-1}(2 t-1+(t-1) q) \cdot \gamma$ non-dominated vertices left.

We can therefore define $D_{3}:=\left\{v \in V(G): v \notin N\left(D_{2}\right)\right\}$ and have that $\left|D_{3}\right| \leqslant k^{t-1}(2 t-1+(t-1) q) \cdot \gamma$, and that $D_{1} \cup D_{2} \cup D_{3}$ is a dominating set of $G$.

We now turn to the proof of the above claim.

Lemma 11. For every vertex $v$ of the graph, the following holds:

$$
\left|\left\{v^{\prime} \in N(v): v^{\prime} \notin N\left(D_{2}\right)\right\}\right|<k^{t-1}(2 t-1+(t-1) q) .
$$


Proof. Assume, for the sake of reaching a contradiction, that there is a vertex $v$ such that $\left|\left\{v^{\prime} \in N(v): v^{\prime} \notin N\left(D_{2}\right)\right\}\right| \geqslant k^{t-1}(2 t-1+(t-1) q)$.

We then define $B_{1}:=\left\{v^{\prime} \in N(v): v^{\prime} \notin N\left(D_{2}\right)\right\}$.

Exactly as in the proof of Lemma 7 , we have that $B_{1}$ can be dominated by at most $k$ elements of $D^{\prime}$. Hence by Lemma 3, we can derive a pseudo-cover $S=\left(u_{1}, \ldots, u_{m}\right)$ of $B_{1}$, where $m \leqslant k$ and every $u_{i}$ is an element of $D^{\prime}$. This leads to the existence of some vertex $u$ in $S$ that covers at least a $1 / k$ fraction of $B_{1} \backslash X$. This yields a vertex $v_{2}$, and a set $B_{2}$.

We can then continue and build a maximal $k$-dominating-sequence $\left(v_{1}, \ldots v_{s}\right)$ of $v$. By construction, this sequence has the property that every $v_{i}$ dominates some elements of $B_{1}$. This is true in particular for $v_{s}$, but also we have that $v_{s} \in D_{2}$, hence a contradiction.

Corollary 2. The graph contains at most $k^{t-1}(2 t-1+(t-1) q) \cdot \gamma$ non-dominated vertices. In particular, the set $D_{3}$ has at most this size.

\section{The algorithm}

In this final section we summarize the algorithm.

1. Compute the set $D_{1}$ of all $v$ such that $N(v)$ cannot be dominated by $2 \nabla_{1}(G)$ vertices different from $v$. Remove $D_{1}$ from $G$ and mark all its neighbors as dominated.

2. In parallel for every vertex $v=v_{1}$ compute all $k$-domination sequences $v_{1}, \ldots, v_{s}$. Add all vertices $v_{s}$ to the set $D_{2}$. Remove $D_{2}$ from $G$ and mark its neighbors as dominated. This is done as follows.

We can learn the neighborhood $N_{2}[v]$ for every vertex $v$ in 2 rounds. In the LOCAL model we can then compute the pseudo-covers without further communication. In two additional rounds can compute the domination sequences from the pseudo-covers (as we need to consider only elements from $N_{2}\left[v_{1}\right]$ ). We report in 2 additional rounds that $v_{s}$ belongs to $D_{2}$ and one more round to mark the neighbors of $D_{2}$ as dominated.

3. In the final round we add all non-dominated vertices to a set $D_{3}$ and return the set $D_{1} \cup D_{2} \cup D_{3}$.

According to Lemma 1, Lemma 10 and Corollary 2 the algorithm computes a $\nabla_{1}+2+3(2 k)^{t(2 k+1)}+k^{t-1}(2 t-1+(t-1) q)$ approximation. This is an absolute constant in $\mathcal{O}\left(\nabla_{1}^{4 t \nabla_{1}+t}\right)$ depending only on $\nabla_{1}(G)$, as also $t<2 \nabla_{1}$.

\section{Conclusion}

We simplified the presentation and generalized the algorithm of Czygrinow et al. [9] from graph classes that exclude some topological minor to graph classes $\mathcal{C}$ where $\nabla_{1}(G)$ is bounded by an absolute constant for all $G \in \mathcal{C}$. This is a property in particular possessed by classes with bounded expansion, which include many commonly studied sparse graph classes. 
It is an interesting and important question to identify the most general graph classes on which certain algorithmic techniques work. The key arguments of Lemma 1 and Lemma 2 work only for classes with $\nabla_{1}(G)$ bounded by an absolute constant. We need different methods to push towards classes with only $\nabla_{0}(G)$ bounded, which are the degenerate classes.

The obtained bounds are still large, but by magnitudes smaller than those obtained in the original work of Czygrinow et al.[9]. It will also be interesting to optimize the algorithm for planar graphs, where additional topological arguments can help to strongly optimize constants and potentially beat the currently best known bound of $52[26,35]$.

\section{References}

1. Akhoondian Amiri, S., Ossona de Mendez, P., Rabinovich, R., Siebertz, S.: Distributed domination on graph classes of bounded expansion. In: Proceedings of the 30th on Symposium on Parallelism in Algorithms and Architectures. pp. 143-151 (2018)

2. Akhoondian Amiri, S., Schmid, S., Siebertz, S.: A local constant factor mds approximation for bounded genus graphs. In: Proceedings of the 2016 ACM Symposium on Principles of Distributed Computing. pp. 227-233 (2016)

3. Amiri, S.A., Schmid, S.: Brief announcement: A log*-time local mds approximation scheme for bounded genus graphs. In: Proc. DISC (2016)

4. Amiri, S.A., Schmid, S., Siebertz, S.: Distributed dominating set approximations beyond planar graphs. ACM Transactions on Algorithms (TALG) 15(3), 1-18 (2019)

5. Bansal, N., Umboh, S.W.: Tight approximation bounds for dominating set on graphs of bounded arboricity. Information Processing Letters 122, 21-24 (2017)

6. Barenboim, L., Elkin, M., Gavoille, C.: A fast network-decomposition algorithm and its applications to constant-time distributed computation. Theoretical Computer Science 751, 2-23 (2018)

7. Brönnimann, H., Goodrich, M.T.: Almost optimal set covers in finite vc-dimension. Discrete \& Computational Geometry 14(4), 463-479 (1995)

8. Czygrinow, A., Hańćkowiak, M., Wawrzyniak, W.: Fast distributed approximations in planar graphs. In: International Symposium on Distributed Computing. pp. 78-92. Springer (2008)

9. Czygrinow, A., Hanckowiak, M., Wawrzyniak, W., Witkowski, M.: Distributed approximation algorithms for the minimum dominating set in k_h-minor-free graphs. In: 29th International Symposium on Algorithms and Computation (ISAAC 2018). Schloss Dagstuhl-Leibniz-Zentrum fuer Informatik (2018)

10. Dinur, I., Steurer, D.: Analytical approach to parallel repetition. In: Proceedings of the forty-sixth annual ACM symposium on Theory of computing. pp. 624-633 (2014)

11. Drange, P.G., Dregi, M.S., Fomin, F.V., Kreutzer, S., Lokshtanov, D., Pilipczuk, M., Pilipczuk, M., Reidl, F., Villaamil, F.S., Saurabh, S., Siebertz, S., Sikdar, S.: Kernelization and sparseness: the case of dominating set. In: 33rd Symposium on Theoretical Aspects of Computer Science, STACS 2016. pp. 31:1-31:14 (2016)

12. Eiben, E., Kumar, M., Mouawad, A.E., Panolan, F., Siebertz, S.: Lossy kernels for connected dominating set on sparse graphs. SIAM Journal on Discrete Mathematics 33(3), 1743-1771 (2019) 
13. Eickmeyer, K., Giannopoulou, A.C., Kreutzer, S., Kwon, O., Pilipczuk, M., Rabinovich, R., Siebertz, S.: Neighborhood complexity and kernelization for nowhere dense classes of graphs. In: 44th International Colloquium on Automata, Languages, and Programming, ICALP 2017, July 10-14, 2017, Warsaw, Poland. pp. 63:1-63:14 (2017)

14. Even, G., Rawitz, D., Shahar, S.M.: Hitting sets when the vc-dimension is small. Information Processing Letters 95(2), 358-362 (2005)

15. Fabianski, G., Pilipczuk, M., Siebertz, S., Torunczyk, S.: Progressive algorithms for domination and independence. In: 36th International Symposium on Theoretical Aspects of Computer Science, STACS 2019, March 13-16, 2019, Berlin, Germany. pp. 27:1-27:16 (2019)

16. Gallager, R.G., Humblet, P.A., Spira, P.M.: A distributed algorithm for minimumweight spanning trees. ACM Transactions on Programming Languages and systems (TOPLAS) 5(1), 66-77 (1983)

17. Garey, M.R., Johnson, D.S.: Computers and intractability, vol. 174. freeman San Francisco (1979)

18. Ghaffari, M., Kuhn, F., Maus, Y.: On the complexity of local distributed graph problems. In: STOC. pp. 784-797. ACM (2017)

19. Har-Peled, S., Quanrud, K.: Approximation algorithms for polynomial-expansion and low-density graphs. SIAM Journal on Computing 46(6), 1712-1744 (2017)

20. Hilke, M., Lenzen, C., Suomela, J.: Brief announcement: local approximability of minimum dominating set on planar graphs. In: Proceedings of the 2014 ACM symposium on Principles of distributed computing. pp. 344-346 (2014)

21. Johnson, D.S.: Approximation algorithms for combinatorial problems. Journal of computer and system sciences 9(3), 256-278 (1974)

22. Jones, M., Lokshtanov, D., Ramanujan, M., Saurabh, S., Suchý, O.: Parameterized complexity of directed steiner tree on sparse graphs. SIAM Journal on Discrete Mathematics 31(2), 1294-1327 (2017)

23. Karp, R.M.: Reducibility among combinatorial problems. In: Complexity of computer computations, pp. 85-103. Springer (1972)

24. Kreutzer, S., Rabinovich, R., Siebertz, S.: Polynomial kernels and wideness properties of nowhere dense graph classes. ACM Transactions on Algorithms (TALG) 15(2), 1-19 (2018)

25. Kuhn, F., Moscibroda, T., Wattenhofer, R.: Local computation: Lower and upper bounds. J. ACM 63(2), 17:1-17:44 (2016)

26. Lenzen, C., Pignolet, Y.A., Wattenhofer, R.: Distributed minimum dominating set approximations in restricted families of graphs. Distributed computing 26(2), 119-137 (2013)

27. Lenzen, C., Wattenhofer, R.: Leveraging linial's locality limit. In: International Symposium on Distributed Computing. pp. 394-407. Springer (2008)

28. Lenzen, C., Wattenhofer, R.: Minimum dominating set approximation in graphs of bounded arboricity. In: International symposium on distributed computing. pp. 510-524. Springer (2010)

29. Lovász, L.: On the ratio of optimal integral and fractional covers. Discrete mathematics 13(4), 383-390 (1975)

30. Nešetřil, J., de Mendez, P.O.: Grad and classes with bounded expansion I. decompositions. European Journal of Combinatorics 29(3), 760-776 (2008)

31. Nešetřil, J., de Mendez, P.O., Wood, D.R.: Characterisations and examples of graph classes with bounded expansion. European Journal of Combinatorics 33(3), 350-373 (2012) 
32. Rozhon, V., Ghaffari, M.: Polylogarithmic-time deterministic network decomposition and distributed derandomization. In: STOC. pp. 350-363. ACM (2020)

33. Siebertz, S.: Greedy domination on biclique-free graphs. Information Processing Letters 145, 64-67 (2019)

34. Wawrzyniak, W.: Brief announcement: a local approximation algorithm for mds problem in anonymous planar networks. In: Proceedings of the 2013 ACM symposium on Principles of distributed computing. pp. 406-408 (2013)

35. Wawrzyniak, W.: A strengthened analysis of a local algorithm for the minimum dominating set problem in planar graphs. Information Processing Letters 114(3), 94-98 (2014) 